\title{
Building Mobile AR Applications using the Outdoor AR Library
}

\author{
Gun A. Lee* and Mark Billinghurst ${ }^{\dagger}$ \\ The Human Interface Technology Laboratory New Zealand, University of Canterbury
}

\begin{abstract}
While early outdoor Augmented Reality (AR) systems required cumbersome equipment to be worn by the user, recent developments in mobile device technology mean that smartphones can now run AR applications. In this tutorial, attendees will learn how to build their own mobile outdoor AR application using the HIT Lab NZ Outdoor AR library. It will start with an overview of outdoor AR technology including core concepts, history and recent research trends. Then we will show how to build a custom mobile AR application using the Outdoor AR library, a component based framework that is easy to customize and reuse.
\end{abstract}

CR Categories: H.5.1 [nformation Interfaces and Presentation]: Multimedia Information Systems-Artificial, augmented, and virtual realities;

Keywords: Mobile outdoor augmented reality, software library

\section{Introduction}

Outdoor Augmented Reality (AR) has been one of the main focus areas for AR research. While early outdoor AR systems required cumbersome equipment to be carried or worn by the user [Feiner et al. 1997; Piekarski et al. 1999], with recent developments in mobile device technology, mainstream smartphones can now run outdoor AR applications. In this tutorial, we introduce recent work in mobile outdoor AR research, and show how to develop a custom mobile outdoor AR application using the Outdoor AR mobile software library.

\section{Part One - Outdoor AR: Concept and Appli- cations}

The first part of the tutorial (approx. $30 \mathrm{~min}$.) will give an overview of outdoor AR technology, introducing key concepts, a brief history, and recent research trends. Attendees will also be presented with user interface design guidelines for outdoor AR experiences. Finally, we will show several mobile outdoor AR applications built using the HIT Lab NZ Outdoor AR library; including CityViewAR [Lee et al. 2012], CCDU 3D, AntarcticAR, and GeoBoids [Lindeman et al. 2012] (see Figure 1).

\section{Part two - Building a Mobile Outdoor AR Application}

In the second part, attendees will learn how to build their own mobile outdoor AR application using the Outdoor AR library (approx. 45 min.). The Outdoor AR library (http://www.hitlabnz.org/mobileAR) is a component based software development framework for easily building mobile outdoor AR applications. It provides a high-level abstraction of user interface and application components common in outdoor AR applications. The components are designed based on the Model-View-

\footnotetext{
*e-mail:gun.lee@hitlabnz.org

†e-mail:mark.billinghurst@hitlabnz.org
}

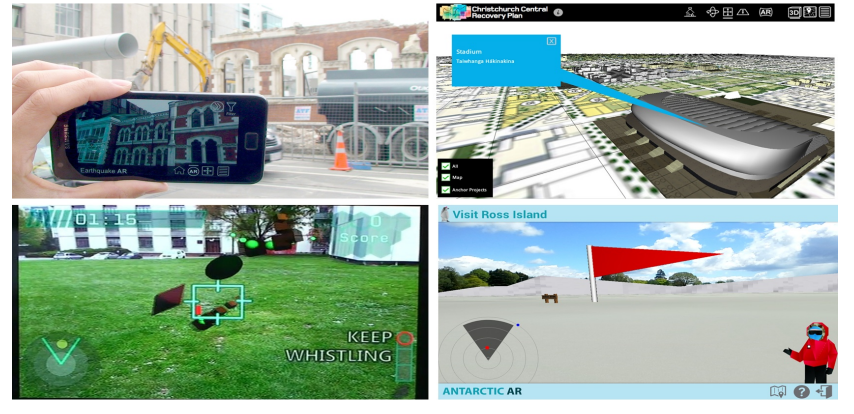

Figure 1: Outdoor AR Applications: CityViewAR, CCDU 3D, Antarctic AR, and GeoBoids (clockwise from top left).

Controller (MVC) design pattern, and by mix and matching and customizing the ready-to-use components, developers can focus on building the domain content, logic and user interface, and spend less time implementing basic functionality. The tutorial will outline the structure of the library and give a step-by-step guide on how to build a mobile outdoor AR application. We will cover how to set up the library, use the components, show 3D models in AR, and add custom information to the scene. It will be easy enough to follow for those who have knowledge of object oriented programming languages (e.g. Java). Although not necessary, previous experience with Android software development will help understanding the concepts. For those who don't have programming skills, we will also briefly introduce how to use a web-based tool to create an outdoor AR scene that can be viewed on a ready-to-use AR browser. By the end of the tutorial, attendees should have the skills necessary to build a basic outdoor AR application.

\section{References}

Feiner, S., Macintyre, B., Hllerer, T., And Webster, A. 1997. A touring machine: Prototyping 3D mobile augmented reality systems for exploring the urban environment. Personal Technologies 1, 4, 208-217.

LeE, G., DÜnser, A., Kim, S., And Billinghurst, M. 2012. CityViewAR: A mobile outdoor AR application for city visualization. In Proc. of IEEE International Symposium on Mixed and Augmented Reality (ISMAR-AMH) 2012, 57-64.

Lindeman, R. W., Lee, G., Beattie, L., Gamper, H., PathiNARUpothi, R., AND Akhilesh, A. 2012. GeoBoids: A mobile AR application for exergaming. In Proc. of IEEE International Symposium on Mixed and Augmented Reality (ISMARAMH) 2012, 93-94.

Piekarski, W., Hepworth, D., Demczuk, V., Thomas, B., AND GUNTHER, B. 1999. A mobile augmented reality user interface for terrestrial navigation. In Proc. 22nd Australasian Computer Science Conference, 122-133. 